\title{
Maintenance avelumab versus continuation of first-line chemotherapy in gastric cancer: JAVELIN Gastric 100 study design
}

\author{
Markus Moehler*,1, Min-Hee Ryu², Mikhail Dvorkin ${ }^{3}$, Keun-Wook Lee ${ }^{4}$, Hasan Ş Coşkun ${ }^{5}$, \\ Rachel Wong ${ }^{6,7}$, Hyun C Chung ${ }^{8}$, Artem Poltoratsky9 ${ }^{9}$ Akihito Tsuji10, Chia Jui Yen ${ }^{11}$, Alina \\ S Muntean ${ }^{12}$, Samuel Le Sourd ${ }^{13}$, Gina M Vaccaro ${ }^{14}$, Lindsay Overton ${ }^{15}$, Narikazu Boku ${ }^{16}$, \\ Zev A Wainberg ${ }^{17}$, Manish Patel ${ }^{18}$, Maitreyi Sharma ${ }^{19}$, Huiling Xiong ${ }^{19}$, Ilaria Conti ${ }^{19}$, \\ Julien Taieb ${ }^{20}$ \& Yung-Jue Bang ${ }^{21}$ \\ ${ }^{1}$ Department of Internal Medicine, Johannes-Gutenberg University, Mainz, Germany \\ 2Department of Oncology, Asan Medical Center, University of Ulsan College of Medicine, Seoul, South Korea \\ ${ }^{3}$ Department of Oncology, BHI of Omsk Region Clinical Oncology Dispensary, Omsk, Russian Federation \\ ${ }^{4}$ Department of Internal Medicine, Seoul National University Bundang Hospital, Seoul National University College of Medicine, \\ Seongnam, South Korea \\ ${ }^{5}$ Department of Medical Oncology, Akdeniz University Medical Faculty, Antalya, Turkey \\ ${ }^{6}$ Department of Medical Oncology, Eastern Health, Box Hill Hospital, Melbourne, VIC, Australia \\ ${ }^{7}$ Monash University, Faculty of Medicine, Nursing and Health Sciences, Melbourne, VIC, Australia \\ ${ }^{8}$ Division of Medical Oncology, Department of Internal Medicine, Yonsei Cancer Center, Yonsei University College of Medicine, \\ Yonsei University Health System, Seoul, South Korea \\ ${ }^{9}$ Department of Oncology, FBI "Scientific Research Institute of Oncology n. a. N. N. Petrov", Russian Federation \\ ${ }^{10}$ Department of Clinical Oncology, Kagawa University Hospital, Kagawa, Japan \\ ${ }^{11}$ Department of Internal Medicine, National Cheng Kung University Hospital, Taiwan, PR China \\ ${ }^{12}$ Department of Research, Oncology Institute "Prof Dr Ion Chiricuta", Cluj Napoca, Romania \\ ${ }^{13}$ Department of Medical Oncology, Centre Eugene-Marquis, Oncologie Médicale, Rennes, France \\ ${ }^{14}$ Division of Hematology and Medical Oncology, Department of Medicine, Oregon Health \& Science University, Portland, OR \\ 97239, USA \\ ${ }^{15}$ Oncology \& Hematology \& Clinical Oncology Research, Confluence Health, Wenatchee, WA 98801, USA \\ ${ }^{16}$ Department of Gastrointestinal Medical Oncology, National Cancer Center Hospital, Tokyo, Japan \\ ${ }^{17}$ Division of Hematology/Oncology, Department of Medicine, UCLA Jonsson Comprehensive Cancer Center, Los Angeles, CA \\ 90095, USA \\ ${ }^{18}$ Department of Medical Oncology, Florida Cancer Specialists/Sarah Cannon Research Institute, Sarasota, FL 34236, USA \\ ${ }^{19} \mathrm{Global}$ Clinical Development Immuno-Oncology, EMD Serono, Billerica, MA 01821, USA \\ ${ }^{20}$ Sorbonne Paris Cité, Paris Descartes University, Department of Gastroenterology \& Digestive Oncology, Georges Pompidou \\ European Hospital, Paris, France \\ ${ }^{21}$ Department of Internal Medicine, Seoul National University College of Medicine, Seoul, South Korea \\ *Author for correspondence: markus.moehler@unimedizin-mainz.de
}

Avelumab is a human anti-PD-L1 IgG1 monoclonal antibody that has shown antitumor activity in early phase studies in advanced/metastatic gastric/gastroesophageal junction cancer, including as first-line maintenance therapy. Here, we describe the design of JAVELIN Gastric 100 (NCT02625610), an open-label, Phase III trial. A total of 499 patients with locally advanced/metastatic HER2- gastric/gastroesophageal junction cancer adenocarcinoma, who had achieved at least stable disease following 12 weeks of first-line oxaliplatin/fluoropyrimidine chemotherapy, have been randomized 1:1 to receive avelumab maintenance therapy or continue chemotherapy. The primary objective is to demonstrate superior overall survival in all randomized patients or in the PD-L1+ population. Secondary objectives are to demonstrate superiority for progression-free survival and objective response rate, compare quality of life measures, and determine safety.

First draft submitted: 30 August 2018; Accepted for publication: 12 October 2018; Published online: 31 October 2018

Keywords: avelumab • gastric cancer • PD-L1 


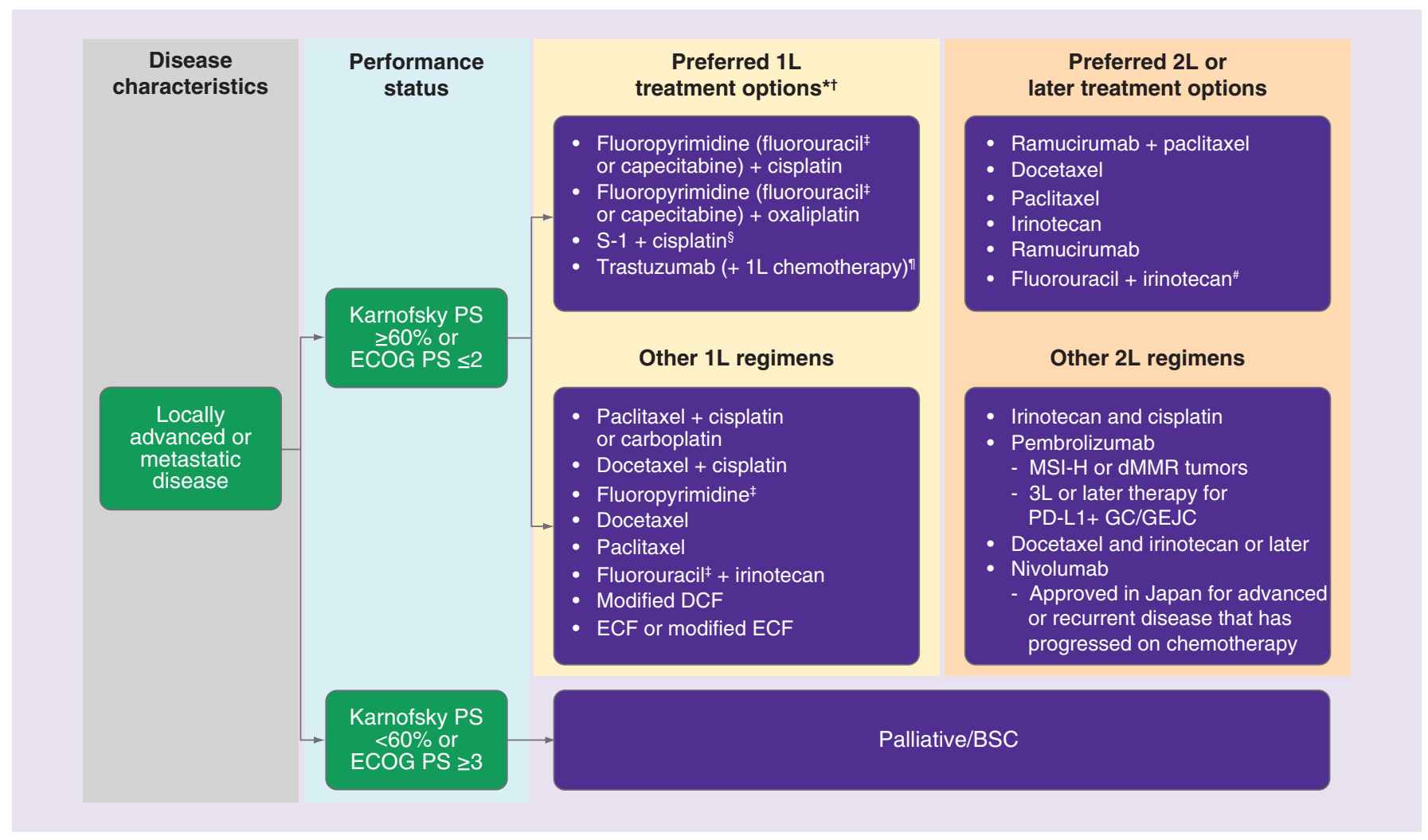

Figure 1. Treatment landscape in advanced gastric/gastroesophageal junction cancer based on National Comprehensive Cancer Network, European Society for Medical Oncology and Japanese guidelines, and recent approvals.

*Participation in clinical trials of new or more tolerable therapy is recommended.

${ }^{\dagger}$ Doublet cytotoxic regimens are preferred because of lower toxicity. Triplet cytotoxic regimens should be reserved for medically fit patients with good PS and access to frequent toxicity evaluation.

¥Leucovorin is indicated with certain fluorouracil-based regimens.

$\S$ Standard-of-care recommendation for $1 \mathrm{~L}$ treatment based on Japanese GC treatment guidelines.

IFor HER2-overexpressing advanced disease.

${ }^{\#}$ Capecitabine may not be used interchangeably with fluorouracil in regimens containing irinotecan and is only recommended if not previously used in $1 \mathrm{~L}$.

1L: First line; 2L: Second line; 3L: Third line; BSC: Best supportive care; DCF: Docetaxel + cisplatin + fluorouracil (substitutions for cisplatin include oxaliplatin or carboplatin); dMMR: Mismatch repair deficient; ECF: Epirubicin, cisplatin and fluorouracil (substitution for cisplatin include use of oxaliplatin, cisplatin or capecitabine); ECOG: Eastern Cooperative Oncology Group; ESMO: European Society for Medical Oncology; GC: Gastric cancer; GEJC: Gastroesophageal junction cancer; MSI-H: Microsatellite instability-high; NCCN: National Comprehensive Cancer Network; PS: Performance status.

Gastric cancer (GC), the fifth most common cancer and the third leading cause of cancer-related death worldwide, presents a considerable global health burden [1]. Although the incidence of GC is decreasing, the most recent estimates indicate that approximately 1 million individuals are diagnosed with GC each year, of whom half are from Asia [1]. In the western hemisphere, most patients with GC are diagnosed at an advanced stage and face a poor prognosis [2], whereas enhanced screening programs in Asia have led to more frequent diagnosis at early stages [3]. Gastroesophageal junction cancer (GEJC) is a distinct cancer from GC, although evidence suggests that GEJC has a similar biology to that of GC, and recommended treatments are similar for both tumor types, particularly in the metastatic setting $[4,5]$.

Systemic chemotherapy is the standard treatment for advanced GC/GEJC [2], and first-line (1L) treatment selection is generally based on patient performance status (PS) and tumor HER2 expression (Figure 1) [3,5-7]. Recommended standard of care for patients with HER2- tumors typically involves doublet cytotoxic regimens including a platinum agent and a fluoropyrimidine $[3,5,6]$. Triplet cytotoxic regimens, which are less well tolerated [8] are generally reserved for fit patients with a good PS [5], although better-tolerated triplet regimens are 
being assessed [9]. For patients with HER2+ tumors, trastuzumab (an anti-HER2 antibody) in combination with cisplatin-based chemotherapy is the standard treatment $[5,10]$. Despite improvements in chemotherapy for patients with advanced GC/GEJC, the median overall survival (OS) remains short, ranging from 8 to 15 months with standard 1L chemotherapy, 4-9 months with second-line (2L) regimens, and 3-5 months with third-line (3L) treatment $[5,11,12]$.

Because patients with advanced GC/GEJC often have poor prognostic factors that limit long-term use of standard combination chemotherapy regimens [3,5,13], an optimal continuum of care, during which all available treatment options are administered to maximize the overall benefit across all lines of treatment, is needed. One strategy that could improve and extend the duration of tumor responses/disease control, progression-free survival (PFS), OS and quality of life (QOL) in the $1 \mathrm{~L}$ setting without additive toxicity is maintenance treatment, which may involve continuation or de-escalation of $1 \mathrm{~L}$ chemotherapy, or sequential treatment with a different agent in patients with nonprogressive disease (switch maintenance) [14-17]. In patients with non-small-cell lung cancer and colorectal cancer, who have a poor prognosis similar to that of advanced GC/GEJC, maintenance treatment has been shown to extend the duration of antitumor benefit following standard $1 \mathrm{~L}$ induction treatment [14-17]. Furthermore, reintroduction of the $1 \mathrm{~L}$ chemotherapy regimen following disease progression on (switch) maintenance treatment is also possible [18]. Although maintenance treatment for advanced GC/GEJC has not been studied extensively, observational and retrospective studies reported to date have shown that maintenance treatment with fluoropyrimidines is feasible and may improve PFS compared with observation [19-21]. Moreover, trastuzumab or ramucirumab are administered continuously until disease progression in patients with GC/GEJC [5], suggesting that evaluation of long-term treatment with other agents is warranted.

Because GC/GEJC is associated with immune system evasion, immunotherapy targeting immunological checkpoint proteins, such as PD-1 and its ligand, PD-L1, which aims to restore immune responses in GC/GEJC, is a rational approach [22-24]. Consistent with this, anti-PD-1 antibodies have shown encouraging efficacy and safety profiles in patients with advanced GC/GEJC [25-28]. In addition, chemotherapy has well-documented immunogenic effects [29], and early clinical trials in various tumor types have shown that combination treatment with immunotherapy and chemotherapy may increase the frequency of antitumor responses compared with chemotherapy alone [29], including in GC/GEJC [26]. The first study to assess whether maintenance immunotherapy could leverage the immunogenic effects of chemotherapy was a recent randomized, Phase II, proof-of-concept trial of maintenance treatment with the anti-CTLA4 antibody ipilimumab after platinum/fluoropyrimidine-based induction chemotherapy in patients with advanced GC/GEJC [30]. Although no significant benefit in median PFS or OS was seen with ipilimumab compared with best supportive care, which included ongoing fluoropyrimidine treatment in approximately $80 \%$ of patients, full interpretation was limited by a relatively short duration of follow-up [30], and the mechanism of action of ipilimumab may not be optimal in the maintenance setting.

\section{Avelumab}

Avelumab (Bavencio ${ }^{\circledR}$ ) is a human anti-PD-L1 IgG1 antibody that binds PD-L1 and inhibits its interaction with PD-1 [31]. Avelumab has shown encouraging efficacy and acceptable safety in patients with various tumors and has received regulatory approval in various countries for the treatment of metastatic Merkel cell carcinoma and in the USA and Canada for advanced urothelial carcinoma that has progressed during or after platinum-containing chemotherapy [32-35]. Two Phase I studies, JAVELIN Solid Tumor (NCT01772004) and JAVELIN Solid Tumor JPN (NCT01943461), have shown that avelumab treatment has an acceptable safety profile and is associated with promising rates of objective response and disease stabilization in patients with advanced GC/GEJC [36,37]. In particular, in the JAVELIN Solid Tumor trial, avelumab was administered to a large Phase IB cohort of 150 patients with locally advanced or metastatic GC/GEJC, who were recruited to two subgroups according to whether or not they had disease progression after $1 \mathrm{~L}$ chemotherapy (i.e., avelumab was administered either as $2 \mathrm{~L}$ therapy after disease progression or as $1 \mathrm{~L}$ maintenance therapy in patients who had achieved at least stable disease [SD]) [36]. The overall response rate (ORR) with avelumab treatment was $6.7 \%$ in both subgroups, but a complete response (CR) was achieved in $2.2 \%$ of patients who received avelumab as $1 \mathrm{~L}$ maintenance treatment. Median OS in the $1 \mathrm{~L}$ maintenance and $2 \mathrm{~L}$ subgroups was 11.1 and 6.6 months, respectively, and in the $1 \mathrm{~L}$ maintenance subgroup, median OS measured from start of $1 \mathrm{~L}$ chemotherapy was 18.7 months. The safety profile of avelumab was generally consistent with that of other anti-PD-L1/PD-1 antibodies and did not overlap with the relatively severe toxicities seen with cytotoxic chemotherapy regimens. Overall, the efficacy and safety of avelumab in the 1L maintenance 
setting seen in the JAVELIN Solid Tumor trial are highly encouraging and warrant evaluation in a larger randomized trial.

\section{JAVELIN Gastric 100 trial}

We describe the design of the randomized, open-label, Phase III trial JAVELIN Gastric 100 (NCT02625610) comparing maintenance avelumab with continuation of $1 \mathrm{~L}$ chemotherapy in patients with advanced GC/GEJC.

\section{Objectives}

The primary objective of this trial is to demonstrate that avelumab maintenance therapy is superior to continuation of $1 \mathrm{~L}$ chemotherapy with regard to OS measured from randomization, that is, the start of maintenance treatment, in all randomized patients or in patients with PD-L1+ tumors. Secondary objectives are to demonstrate superior PFS and ORR with avelumab versus continuation of $1 \mathrm{~L}$ chemotherapy (according to Response Evaluation Criteria in Solid Tumors v1.1 and as per investigator assessment, with baseline defined as the last measurement performed prior to randomization), to evaluate the safety and tolerability of avelumab, and to assess patient-reported outcomes (PROs) and QOL measures with avelumab versus continuation of $1 \mathrm{~L}$ chemotherapy. Exploratory objectives include evaluation of target lesion reduction from baseline during the maintenance phase; exploration of the molecular, cellular and soluble markers relevant to the mechanism of action of avelumab and the response or resistance to treatment, including PD-L1 expression in tumor cells and immune infiltrates; assessment of the duration of and time to response with avelumab; and characterization of the pharmacokinetic profile and immunogenicity of avelumab.

\section{Key eligibility criteria}

All patients must be aged $\geq 18$ years and have histologically confirmed, previously untreated, unresectable, locally advanced or metastatic HER2- GC/GEJC measurable by Response Evaluation Criteria in Solid Tumors v1.1. Other inclusion criteria are: Eastern Cooperative Oncology Group PS score of 0-1; availability of a formalinfixed, paraffin-embedded block containing tumor tissue or unstained tumor slides suitable for PD-L1 expression assessment; estimated life expectancy of $\geq 12$ weeks; adequate renal, hepatic and hematologic function; no major surgery within 4 weeks of enrollment (other than diagnostic biopsy); and no vaccination within 55 days of the first dose of avelumab (with exception for inactivated vaccines). Patients are not permitted to have received any prior chemotherapy for advanced/metastatic disease or any prior therapy with a drug targeting a T-cell co-regulatory protein, and are not permitted to receive concurrent treatment with anticancer or immunosuppressive agents. Additionally, enrolled patients must have no evidence of CNS metastases or malignant disease other than GC within the previous 5 years and no history of uncontrolled or clinically significant illness, including prior organ transplant, infection, autoimmune disease and cardiovascular disease. Finally, patients must have no history of allergy to monoclonal antibodies, no history of anaphylaxis, and no uncontrolled asthma.

\section{Study design}

JAVELIN Gastric 100 is a randomized, open-label, global, multicenter, Phase III trial. Enrollment began in December 2015 and is now complete. Patients were recruited at 200 sites in North America, South America, Asia-Pacific and Europe. All recruited patients received 1L therapy with oxaliplatin, leucovorin and 5-fluorouracil (FOLFOX/FLO) or oxaliplatin and capecitabine (XELOX/CAPOX) for 12 weeks (Figure 2). Following this induction phase, patients were reassessed for eligibility, and 499 patients with a CR, partial response or SD were randomized 1:1 to receive maintenance therapy with either avelumab $10 \mathrm{mg} / \mathrm{kg}$ administered by intravenous infusion every 2 weeks or continuation of the same chemotherapy regimen from the induction phase. Patients randomized to the nonavelumab arm deemed ineligible to receive further chemotherapy receive best supportive care alone. Patients randomized to receive avelumab must be premedicated with an antihistamine and paracetamol/acetaminophen (modified based on local treatment standards and guidelines as appropriate) 30-60 min prior to the first four infusions. Maintenance treatment is given until confirmed disease progression, significant clinical deterioration, unacceptable toxicity or other protocol-specified criteria for discontinuation occur. The primary end point of the trial is OS. Secondary end points include PFS, best overall response, PROs/QOL measures, safety and tumor biomarkers. 


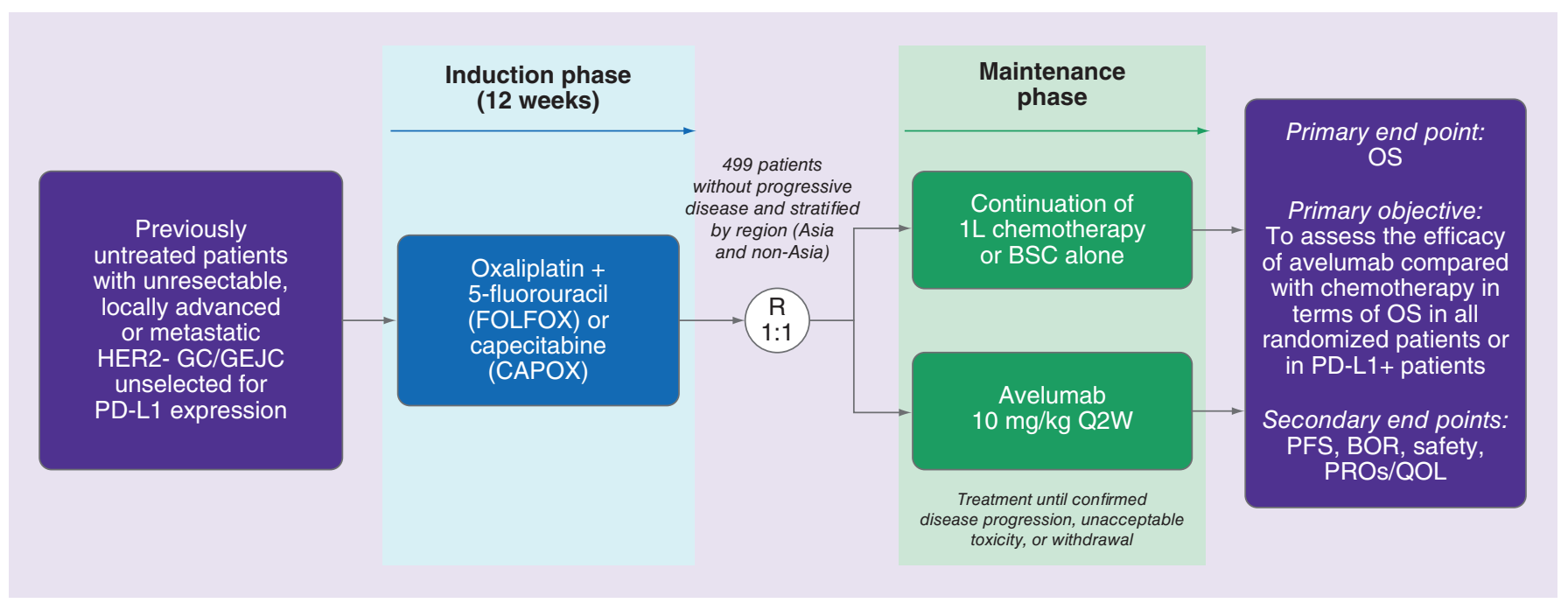

Figure 2. Gastric 100 trial design.

1L: First line; BOR: Best overall response; BSC: Best supportive care; CAPOX: Capecitabine and oxaliplatin; FOLFOX: Oxaliplatin, leucovorin and fluorouracil; GC: Gastric cancer; GEJC: Gastroesophageal junction cancer; OS: Overall survival; PFS: Progression-free survival; PRO: Patient-reported outcome; Q2W: Every 2 weeks; QOL: Quality of life; R: Randomization.

\section{Evaluations}

Antitumor responses are assessed via computed tomography or MRI every 6 weeks for the first 12 months after randomization and every 12 weeks thereafter. Safety is assessed throughout and is graded based on National Cancer Institute Common Terminology Criteria for Adverse Events v4.03. Treatment-related adverse events are monitored for $30( \pm 5)$ days following the end of protocol treatment. Potential immune-related adverse events that may result from avelumab treatment are considered adverse events of special interest. Blood samples are collected to analyze the pharmacokinetic profile and immunogenicity of avelumab. PROs and QOL are assessed using the European Organisation for Research and Treatment of Cancer (EORTC) QLQ-C30, EuroQoL EQ5D-5 L and EORTC Gastric Cancer Module QLQ-STO22 questionnaires. PD-L1 expression in tumor cells and/or cells in the tumor microenvironment (nonmalignant tumor-infiltrating cells and stromal cells) will be assessed retrospectively using the PD-L1 IHC 73-10 assay (Dako, CA, USA; under license of Merck KGaA), an investigational immunohistochemistry assay developed for the Dako Autostainer Link 48 platform, which uses the 73-10 anti-PD-L1 monoclonal antibody clone. PD-L1 expression will be scored based on the percentage of PD-L1+ cells and the intensity of staining. Other predictive biomarker assessments are also planned.

\section{Statistical analysis methods}

Efficacy end points are analyzed in all randomized patients on an intent-to-treat basis. The primary analysis population for all analyses of efficacy and safety consists of all patients who have an overall tumor response status of $\mathrm{CR}$, partial response or SD after completing the induction phase and who are subsequently randomized to trial treatment. The sample size of this trial is driven by the primary end point of OS, which is defined as the time from randomization to the date of death due to any cause. This trial has two primary hypotheses, which are that avelumab is superior to $1 \mathrm{~L}$ chemotherapy in terms of OS in all randomized patients and in the PD-L1+ population. Superiority will be tested with a hierarchical strategy that preserves the type 1 error rate at $2.5 \%$ (one sided). The study will be considered positive if at least one of the primary hypotheses is statistically significant in either of the populations. OS will be summarized using the Kaplan-Meier method, and 95\% CIs for the medians will be calculated using the two-sided Brookmeyer and Crowley method. Based on a hierarchical testing strategy, PFS and best overall response will be tested at the primary analysis only if both OS hypotheses (superiority in all randomized patients and in PD-L1+ patients) reach statistical significance. All safety and QOL measures will be assessed using descriptive statistics by treatment arm. 


\section{Ethical considerations}

The JAVELIN Gastric 100 trial is being conducted in compliance with the protocol, in accordance with the ethical principles put forward in the second Declaration of Helsinki, and in accordance with good clinical practice rules. All patients provided written informed consent.

\section{Discussion \& future perspective}

Across several studies, anti-PD-L1/PD-1 antibodies have shown clinical activity and durability of response in pretreated patients with advanced GC/GEJC and disease progression following chemotherapy [25-28,36-38], leading to regulatory approval of nivolumab in Japan for patients with advanced GC progressed after chemotherapy (based on a Phase III trial vs placebo) $[27,39]$, and accelerated approval of pembrolizumab in the USA as $3 \mathrm{~L}$ treatment for advanced PD-L1+ GC/GEJC (based on a large, Phase I, single-arm cohort) [26,40]. However, it has recently been reported that two randomized, Phase III trials of later-line treatment for GC/GEJC did not show superior OS with an anti-PD-1 or anti-PD-L1 antibody when compared directly with chemotherapy; this includes KEYNOTE-061 (NCT02370498), a trial of 2L pembrolizumab versus paclitaxel [41], and JAVELIN Gastric 300 (NCT02625623), a trial of $3 \mathrm{~L}$ avelumab versus physician's choice of chemotherapy [42]. These findings indicate that anti-PD-L1/PD-1 monotherapy is not superior to chemotherapy in patients with disease progression on prior chemotherapy, increasing the interest in Phase III trials of GC/GEJC assessing alternative immunotherapy-based strategies incorporating sequential or combination chemotherapy.

Anti-PD-1 agents have shown encouraging results in early phase trials when administered as monotherapy or in combination with chemotherapy for $1 \mathrm{~L}$ treatment of advanced GC/GEJC. In this setting, standard chemotherapy is associated with response rates of $17-52 \%$ [5]. In two cohorts of patients with GC/GEJC enrolled in the KEYNOTE-059 study, the ORR with $1 \mathrm{~L}$ pembrolizumab combined with 5-fluorouracil and cisplatin was $60 \%$ and with $1 \mathrm{~L}$ pembrolizumab monotherapy (patients with PD-L1+ tumors only) was $26 \%$ [43]. In addition, interim analyses from ATTRACTION-04, an ongoing Phase II/III trial assessing combination treatment with nivolumab and chemotherapy as $1 \mathrm{~L}$ treatment for advanced GC/GEJC, showed ORRs of 67 and $71 \%$ in patients treated with nivolumab plus either S-1 plus oxaliplatin or capecitabine and oxaliplatin [44].

Maintenance treatment with anti-PD-L1/PD-1 antibodies after $1 \mathrm{~L}$ induction chemotherapy may provide an alternative treatment strategy to $1 \mathrm{~L}$ combination treatment, with the aim of enabling patients with advanced GC/GEJC to obtain the efficacy benefits of checkpoint inhibitor therapy without the increased toxicity burden of combination treatment. This treatment strategy might also better preserve patient QOL and enable a longer duration of treatment benefit. To date, JAVELIN Gastric 100 is the only Phase III trial evaluating checkpoint inhibitor maintenance treatment in advanced GC/GEJC, and results from this trial will determine whether avelumab monotherapy as maintenance treatment has the potential to improve OS in all randomized patients or in PD-L1+ patients, achieve a more tolerable safety profile and improve patient QOL compared with continuation of $1 \mathrm{~L}$ chemotherapy. Durvalumab, an engineered human IgG1 anti-PD-L1 antibody, is also being tested in the maintenance setting in an ongoing Phase II study (PLATFORM; NCT02678182), although data have not yet been reported.

The ideal strategy for incorporating immunotherapy in the continuum of care for patients with advanced GC/GEJC remains to be defined. The JAVELIN Gastric 100 trial of avelumab, along with Phase III trials of other agents in different lines/populations, will provide further information on the role of anti-PD-L1/PD-1 therapies in the treatment landscape for GC/GEJC. It is possible that in the future, physicians may tailor treatment strategies based on efficacy, toxicity, QOL considerations and potential impact on subsequent treatment options. This could include the choice of administering anti-PD-1 or anti-PD-L1 therapy in the $1 \mathrm{~L}$ setting as monotherapy, maintenance therapy or in combination with chemotherapy or another immunotherapy. Furthermore, in patients with disease progression following immunotherapy-containing maintenance regimens for GC/GEJC, it may be possible to reintroduce previously administered chemotherapy. Results from the JAVELIN Gastric 100 trial and other ongoing Phase III trials are eagerly awaited to help define future treatment for patients with advanced GC/GEJC. 


\section{Background}

- Combination cytotoxic chemotherapy is the foundation of first-line (1L) treatment for patients with advanced gastric/gastroesophageal junction cancer (GC/GEJC), although prognosis with standard regimens remains poor.

- Immunotherapy targeting the PD-1/PD-L1 interaction has been associated with clinical activity in patients with GC/GEJC, but its optimal role within the continuum of care remains unclear.

- Maintenance therapy is an established strategy for treatment of various advanced tumors based on studies showing prolonged antitumor responses and extended remissions.

- No randomized studies reported to date have evaluated maintenance treatment for patients with advanced GC/GEJC.

\section{Avelumab}

- Avelumab is a human IgG1 anti-PD-L1 monoclonal antibody that has shown durable clinical activity in a range of tumors, and is approved in various countries worldwide for the treatment of metastatic Merkel cell carcinoma and in the USA and Canada for advanced urothelial carcinoma that has progressed during or after platinum-containing chemotherapy.

- In a Phase IB cohort, which enrolled 150 patients with advanced or metastatic GC/GEJC in two subgroups who received avelumab as $1 \mathrm{~L}$ maintenance or second-line therapy, avelumab was well tolerated and showed promising clinical activity; in particular, in the $1 \mathrm{~L}$ maintenance subgroup, the overall response rate was $6.7 \%$ (complete response in 2.2\%), and median OS was 11.1 months measured from the start of avelumab therapy and 18.7 months measured from start of $1 \mathrm{~L}$ chemotherapy.

\section{JAVELIN Gastric 100 trial}

- JAVELIN Gastric 100 is a randomized, open-label, Phase III trial.

- Eligible patients have unresectable, measurable, locally advanced or metastatic HER2- GC/GEJC adenocarcinoma without prior treatment for advanced disease and are not preselected based on PD-L1 expression.

- Following 12 weeks of $1 \mathrm{~L}$ oxaliplatin/fluoropyrimidine chemotherapy, 499 patients achieving stable disease, partial response or complete response were randomized 1:1 to receive maintenance therapy with either avelumab or continuation of the same $1 \mathrm{~L}$ chemotherapy regimen until disease progression, unacceptable toxicity or withdrawal.

- The primary end point is OS, assessed in all randomized patients and in those with PD-L1+ tumors.

- This study will reveal potential treatment strategies for patients with advanced GC/GEJC by providing the first evaluation of anti-PD-L1 maintenance therapy in this setting.

Supplementary data

An infographic accompanies this paper at the end of the references section. To download the infographic that accompanies this paper, please visit the journal website at: www.futuremedicine.com/doi/full/10.2217/fon-2018-0668

\section{Acknowledgements}

The authors would like to thank the patients and their families, investigators, co-investigators, and the study teams at each of the participating centers.

Financial \& competing interests disclosure

The JAVELIN Gastric 100 trial discussed in this manuscript is sponsored by Merck KGaA and is part of an alliance between Merck KGaA and Pfizer (New York, NY, USA). M Moehler has acted in a consultant/advisory role and has provided speaker services for Amgen, Bayer, Merck \& Co, Merck KGaA and Roche, and has received travel expenses from Amgen, Merck KGaA and Pfizer. M-H Ryu has acted in a consultant/advisory role for and received honoraria from Bayer, Bristol-Myers Squibb, DAE HWA Pharmaceutical, Eli Lilly, Novartis, Ono Pharmaceutical, Pfizer, Roche, Sanofi and Taiho, and has received research grants from Novartis. K-W Lee's institution has received research grants from AstraZeneca, Merck \& Co, Ono Pharmaceutical, Roche and Taiho Pharmaceutical. $\mathrm{R}$ Wong has received travel expenses from AstraZeneca. HC Chung has acted in a consultant/advisory role for Bristol-Myers Squibb, Celltrion Healthcare, Eli Lilly, Merck \& Co, Merck KGaA, Quintiles and Taiho, has provided speaker services for Eli Lilly, Foundation Medicine and Merck KGaA, and has received research grants from Bristol-Myers Squibb, Eli Lilly, GlaxoSmithKline, Merck \& Co, Merck KGaA, Ono Pharmaceutical and Taiho Pharmaceutical. A Tsuji has received honoraria from Bristol-Myers Squibb, Chugai Pharma, Daiichi Sankyo, Merck KGaA, Taiho Pharmaceutical and Takeda, and has provided speaker services for Chugai Pharma, Merck KGaA, Taiho Pharmaceutical and Takeda. AS Muntean has received travel expenses from Janssen, Merck KGaA and Roche. GM Vaccaro has acted in a consultant/advisory role for Genentech and Taiho Pharmaceutical, and has received research funding from Celgene. N Boku has received honoraria from Chugai Pharma, Eli Lilly, Merck KGaA, Ono Pharmaceutical, Shionogi Pharma, Taiho Pharmaceutical and Yakult Honsha, and his institution has received research grants from Bristol-Myers Squibb, Ono 
Pharmaceutical and Taiho Pharmaceutical. M Sharma, H Xiong and I Conti are employees of EMD Serono, a company of Merck KGaA, Darmstadt, Germany. J Taieb has acted in a consultant/advisory role for Amgen, Baxalta, Celgene, Eli Lilly, Merck KGaA, Roche/Genentech, and Servier, and has provided speaker services for Amgen, Baxalta, Eli Lilly, Merck KGaA, Roche/Genentech, Sanofi, and Servier. Y-J Bang has acted in a consultant/advisory role for Merck KGaA, and his institution has received research grants from Merck KGaA. M Dvorkin, HS Coşkun, A Poltoratsky, CJ Yen, S Le Sourd, L Overton, ZA Wainberg and M Patel have no competing interests. The authors have no other relevant affiliations or financial involvement with any organization or entity with a financial interest in or financial conflict with the subject matter or materials discussed in the manuscript apart from those disclosed.

Medical writing support was provided by Clinical Thinking and funded by Merck KGaA and Pfizer.

\section{Open access}

This work is licensed under the Attribution-NonCommercial-NoDerivatives 4.0 Unported License. To view a copy of this license, visit http://creativecommons.org/licenses/by-nc-nd/4.0/

\section{References}

Papers of special note have been highlighted as: $\bullet$ of interest; $\bullet \bullet$ of considerable interest

1. Ferlay J, Soerjomataram I, Dikshit R et al. Cancer incidence and mortality worldwide: sources, methods and major patterns in GLOBOCAN 2012. Int. J. Cancer 136(5), e359-e386 (2015).

2. Kothari N, Almhanna K. Current status of novel agents in advanced gastroesophageal adenocarcinoma. J. Gastrointest. Oncol. 6, 60-74 (2015).

3. Smyth EC, Verheij M, Allum W et al. Gastric cancer: ESMO clinical practice guidelines for diagnosis, treatment and follow-up. Ann Oncol. 27(Suppl. 5), v38-v49 (2016).

4. Buas M, Vaughan TL. Epidemiology and risk factors for gastroesophageal junction tumors: understanding the rising incidence of this disease. Semin. Radiat. Oncol. 23(1), 3-9 (2013).

5. National Comprehensive Cancer Network. NCCN clinical practice guidelines in oncology: gastric cancer. V2.2018. www.nccn.org/professionals/physician_gls/def ault.aspx

6. Japanese Gastric Cancer Association. Japanese gastric cancer treatment guidelines 2014 (ver.4). Gastric Cancer 20(1), 1-19 (2017).

7. Jou E, Rajdev L. Current and emerging therapies in unresectable and recurrent gastric cancer. World J. Gastroenterol. 22(20), 4812-4823 (2016).

8. Bittoni A, Del Prete M, Scartozzi M et al. Three drugs vs two drugs first-line chemotherapy regimen in advanced gastric cancer patients: a retrospective analysis. Springerplus 4, 743 (2015).

9. Al-Batran S, Pauligk C, Homann N et al. Docetaxel, oxaliplatin, and fluorouracil/leucovorin (FLOT) for resectable esophagogastric cancer: updated results from multicenter, randomized Phase 3 FLOT4-AIO trial (German Gastric Group at AIO). Ann. Oncol. 28(Suppl. 5), abstract LBA27_PR (2017).

10. Boku N. HER2-positive gastric cancer. Gastric Cancer 17(1), 1-12 (2014).

11. Kim SM, Park SH. Chemotherapy beyond second-line in advanced gastric cancer. World J. Gastroenterol. 21(29), 8811-8816 (2015).

12. Chan WL, Yuen KK, Siu SW, Lam KO, Kwong DL. Third-line systemic treatment versus best supportive care for advanced/metastatic gastric cancer: a systematic review and meta-analysis. Crit. Rev. Oncol. Hematol. 116, 68-81 (2017).

13. Wagner AD, Syn NL, Moehler M et al. Chemotherapy for advanced gastric cancer. Cochrane Database Syst. Rev. 8, CD004064 (2017).

14. Tournigand C, Chibaudel B, Samson B et al. Bevacizumab with or without erlotinib as maintenance therapy in patients with metastatic colorectal cancer (GERCOR DREAM; OPTIMOX3): a randomised, open-label, Phase 3 trial. Lancet Oncol. 16(15), 1493-1505 (2015).

- A Phase III trial showing significant efficacy with a maintenance strategy in patients with metastatic colorectal cancer.

15. Simkens LH, van Tinteren H, May A et al. Maintenance treatment with capecitabine and bevacizumab in metastatic colorectal cancer (CAIRO3): a Phase 3 randomised controlled trial of the Dutch Colorectal Cancer Group. Lancet 385(9980), 1843-1852 (2015).

- A Phase III trial showing significant efficacy with a different maintenance regimen in patients with metastatic colorectal cancer.

16. Antonia SJ, Villegas A, Daniel D et al. Durvalumab after chemoradiotherapy in stage III non-small-cell lung cancer. N. Engl. J. Med. 377(20), 1919-1929 (2017).

-• First randomized Phase III trial to show significantly improved efficacy with a checkpoint inhibitor used as a sequential treatment (similar to maintenance treatment).

17. Ciuleanu T, Brodowicz T, Zielinski C et al. Maintenance pemetrexed plus best supportive care versus placebo plus best supportive care for non-small-cell lung cancer: a randomised, double-blind, Phase 3 study. Lancet 374(9669), 1432-1440 (2009).

18. Kuczynski EA, Sargent DJ, Grothey A, Kerbel RS. Drug rechallenge and treatment beyond progression - implications for drug resistance. Nat. Rev. Clin. Oncol. 10(10), 571-587 (2013). 
19. Meulendijks D, de Groot JW, Los M et al. Bevacizumab combined with docetaxel, oxaliplatin, and capecitabine, followed by maintenance with capecitabine and bevacizumab, as first-line treatment of patients with advanced HER2-negative gastric cancer: a multicenter Phase 2 study. Cancer 122(9), 1434-1443 (2016).

20. Eren OO, Ozturk MA, Sonmez OU, Oyan B. Safety, feasibility, and efficacy of capecitabine maintenance in patients with advanced gastric cancer: a retrospective study. Am. J. Ther. 23(6), e1493-e1497 (2016).

21. Li W, Zhao X, Wang H et al. Maintenance treatment of uracil and tegafur (UFT) in responders following first-line fluorouracil-based chemotherapy in metastatic gastric cancer: a randomized Phase 2 study. Oncotarget 8(23), 37826-37834 (2017).

22. Buchbinder EI, Desai A. CTLA-4 and PD-1 pathways: similarities, differences, and implications of their inhibition. Am. J. Clin. Oncol. 39(1), 98-106 (2016).

23. Postow MA, Callahan MK, Wolchok JD. Immune checkpoint blockade in cancer therapy. J. Clin. Oncol. 33(17), 1974-1982 (2015).

24. Alsina M, Moehler M, Hierro C, Guardeño R, Tabernero J. Immunotherapy for gastric cancer: a focus on immune checkpoints. Target. Oncol. 11(4), 469-477 (2016).

25. Muro K, Chung HC, Shankaran V et al. Pembrolizumab for patients with PD-L1-positive advanced gastric cancer (KEYNOTE-012): a multicentre, open-label, Phase 1B trial. Lancet Oncol. 17(6), 717-726 (2016).

26. Fuchs CS, Doi T, Jang RW et al. Safety and efficacy of pembrolizumab monotherapy in patients with previously treated advanced gastric and gastroesophageal junction cancer: Phase 2 clinical KEYNOTE-059 trial. JAMA Oncol. 4(5), e180013 (2018).

27. Kang YK, Boku N, Satoh T et al. Nivolumab in patients with advanced gastric or gastro-oesophageal junction cancer refractory to, or intolerant of, at least two previous chemotherapy regimens (ONO-4538-12, ATTRACTION-2): a randomised, double-blind, placebo-controlled, Phase 3 trial. Lancet 390, 2461-2471 (2017).

28. Chau I, Chen LT, Kang YK et al. Nivolumab safety profile in Asian and western patients with chemotherapy-refractory (CTx-R) advanced gastric/gastroesophageal junction (adv G/GEJ) cancer from the ATTRACTION-2 and CheckMate-032 trials. J. Clin. Oncol. 36(Suppl. 4S), abstract 90 (2018).

29. Hato SV, Khong A, de Vries IJ, Lesterhuis WJ. Molecular pathways: the immunogenic effects of platinum-based chemotherapeutics. Clin. Cancer Res. 20, 2831-2837 (2014).

30. Bang YJ, Cho JY, Kim YH et al. Efficacy of sequential ipilimumab monotherapy vs best supportive care for unresectable locally advanced/metastatic gastric or gastroesophageal junction cancer. Clin. Cancer Res. 23, 5671-5678 (2017).

- Randomized Phase II trial assessing switch-maintenance therapy with an anti-CTLA-4 checkpoint inhibitor in gastric/gastroesophageal junction cancer (GC/GEJC).

31. Heery CR, O'Sullivan-Coyne G, Madan RA et al. Avelumab for metastatic or locally advanced previously treated solid tumours (JAVELIN solid tumor): a Phase 1A, multicohort, dose-escalation trial. Lancet Oncol. 18, 587-598 (2017).

32. Bavencio (avelumab) injection, package insert. Merck KGaA, Darmstadt, Germany (2017).

33. Bavencio (avelumab) injection, summary of product characteristics. Merck KGaA, Darmstadt, Germany (2017).

34. Bavencio (avelumab) injection, prescribing information (Japan). Merck KGaA, Darmstadt, Germany (2017).

35. Swissmedic authorisation for Bavencio (avelumab). www.swissmedic.ch/swissmedic/en/home/humanarzneimittel/authorisations/authoris ed-medicinal-products-with-new-active-substances/bavencio_konzentrat_zur_herstellung_einer_inf usionsloesung_avelumabum.html

36. Chung HC, Arkenau HT, Lee J et al. Avelumab (anti-PD-L1) as first-line maintenance or second-line therapy in patients with advanced gastric or gastroesophageal junction cancer: updated Phase 1B results from the JAVELIN Solid Tumor trial. Cancer Res. 78(13 Suppl.), CT111 (2018).

-• Single-arm Phase IB study showing promising efficacy for avelumab administered as switch-maintenance treatment in patients with advanced GC/GEJC, which supported the rationale for the JAVELIN Gastric 100 Phase III trial.

37. Hironaka S, Shitara K, Iwasa S et al. Avelumab (MSB0010718C; anti-PD-L1) in Japanese patients with advanced gastric cancer: results from a Phase 1B trial. Ann. Oncol. 27(Suppl. 7), abstract 02-10-4 (2016).

38. Bang YJ, Golan T, Lin CC et al. Interim safety and clinical activity in patients (pts) with locally advanced and unresectable or metastatic gastric or gastroesophageal junction (G/GEJ) adenocarcinoma from a multicohort Phase 1 study of ramucirumab (R) plus durvalumab (D). J. Clin. Oncol. 36(Suppl. 4S), abstract 92 (2018).

39. Bristol-Meyers Squibb Company. Japan Ministry of Health, Labor and Welfare approves Opdivo (nivolumab) for the treatment of patients with unresectable advanced or recurrent gastric cancer which has progressed after chemotherapy. Press release: https://news.bms.com/press-release/corporatefinancial-news/japan-ministry-health-labor-and-welfare-approves-opdivo-nivolu

40. Keytruda (pembrolizumab), package insert. Merck \& Co, Inc, Whitehouse Station, USA (2017).

41. Shitara K, Özgüroğlu M, Bang YJ et al. Pembrolizumab versus paclitaxel for previously treated, advanced gastric or gastro-oesophageal junction cancer (KEYNOTE-061): a randomised, open-label, controlled, Phase 3 trial. Lancet 392(10142), 123-133 (2018).

- Phase III trial showing that pembrolizumab (anti-PD-1) had similar efficacy to paclitaxel chemotherapy (no improvement) in patients with advanced GC/GEJC who had disease progression on prior chemotherapy. 
42. Bang Y-J, Yañez Ruiz E, Van Cutsem E et al. Phase 3, randomised trial of avelumab versus physician's choice of chemotherapy as third-line treatment for patients with advanced gastric or gastro-oesophageal junction cancer: primary analysis of JAVELIN Gastric 300. Ann. Oncol. 29(10), 2052-2060 (2018).

- Phase III trial showing that avelumab (anti-PD-L1) had similar efficacy to chemotherapy (no improvement) as third-line treatment for advanced GC/GEJC.

43. Wainberg ZA, Jalal S, Muro K et al. KEYNOTE-059 update: efficacy and safety of pembrolizumab alone or in combination with chemotherapy in patients with advanced gastric or gastroesophageal (G/GEJ) cancer. Ann. Oncol. 28(Suppl. 5), abstract LBA28 (2017)

44. Kang Y, Kato K, Chung HC et al. Interim safety and clinical activity of nivolumab (nivo) in combination with S-1/capecitabine plus oxaliplatin in patients(pts) with previously untreated unresectable advanced or recurrent gastric/gastroesophageal junction (G/GEJ) cancer: part 1 study of ATTRACTION-04 (ONO-4538-37). Ann. Oncol. 28(Suppl. 5), abstract 671P (2017). 


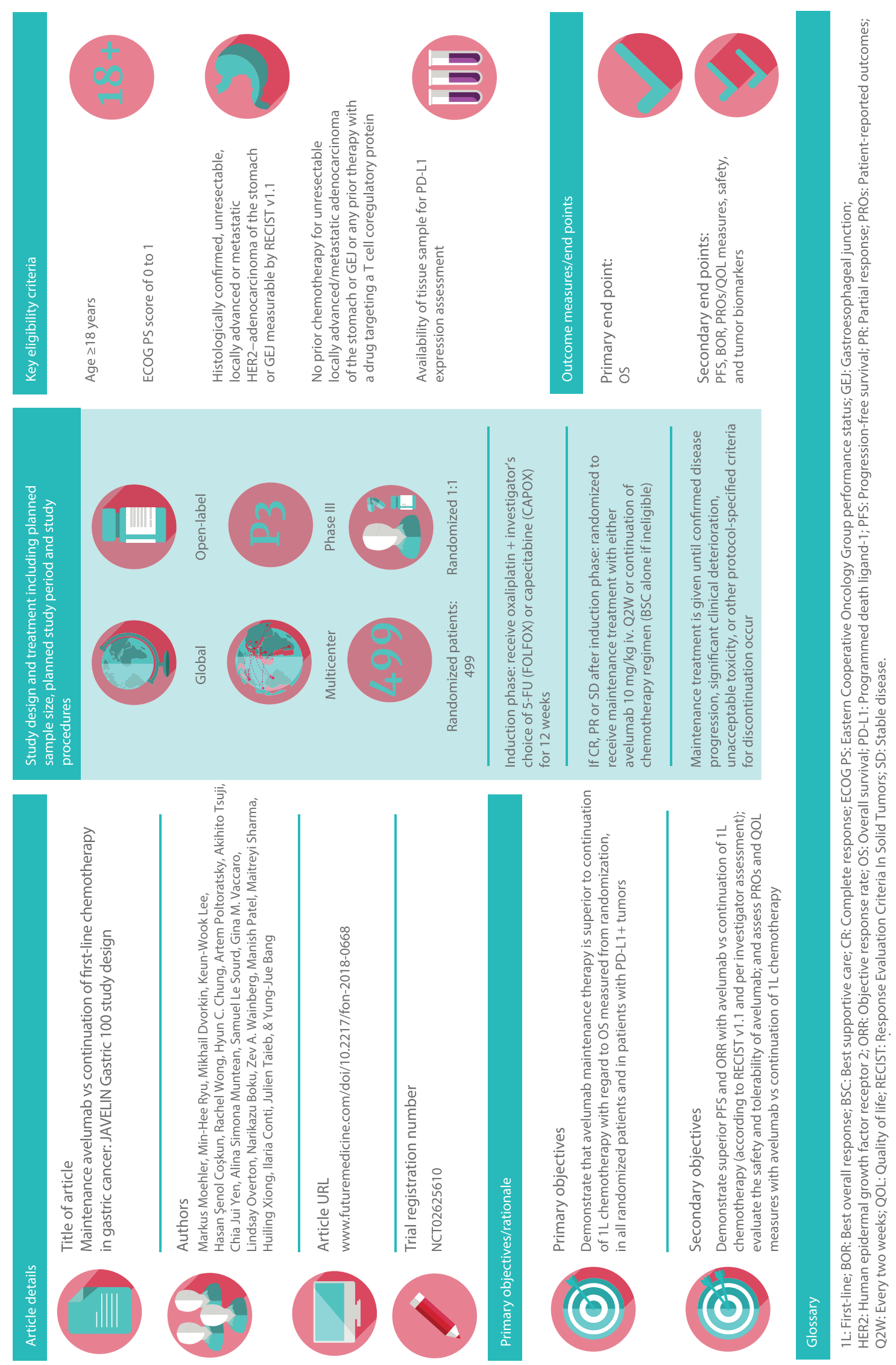

